\title{
Using partially ordered sets to represent and predict true patterns of gene response to treatments
}

\author{
Nam S Vo, Vinhthuy Phan ${ }^{*}$ \\ From 12th Annual UT-ORNL-KBRIN Bioinformatics Summit 2013 \\ Buchanan, TN, USA. 22-24 March 2013
}

\section{Background}

Advances in biotechnology have empowered highthroughput measurement of gene expression levels for tens of thousands of genes simultaneously. This means that one sample size must be used for all genes in most experimental designs [1,2], which implies that patterns of response of highly variantly expressed genes might not be measured accurately. Response patterns of gene expression data with multiple treatments have been characterized using post hoc pairwise comparisons by several researchers $[3,4]$. Nevertheless, these researchers did not address how to cope with highly variantly expressed genes with inaccurate patterns due to having too few experimental samples.

\section{Results}

We show that dependencies of pairwise comparison outcomes in post hoc calculations can be exploited to infer true response patterns of genes with inaccurate patterns due to having too few experimental samples.

Characterizing such response patterns as partially ordered sets, we show that linearly orderable patterns are more likely true patterns and those that are not linearly orderable cannot be true patterns. We propose a strategy to predict most likely linearly orderable extensions of such patterns. Using microarray data of rats' liver cells, we showed that this approach yielded more and better functionally enriched gene lists than a conventional approach.

\section{Conclusions}

This approach opens up opportunities to design costeffective experiments, in which only a conservatively large sample size is needed to collect expression levels of almost all genes. For most genes, such a sample size is sufficient. For highly variantly expressed genes, our method can help infer true response patterns.

Published: 22 October 2013

\section{References}

1. Lee ML, Kuo FC, Whitmore GA, Sklar J: Importance of replication in microarray gene expression studies: statistical methods and evidence from repetitive CDNA hybridization. Proc Natl Acad Sci 2000, 97(18):9834-9839.

2. Glaus P, Honkela A, Rattray M: Identifying differentially expressed transcripts from RNA-seq data with biological variation. Bioinformatics 2012, 28(13):1721-1728.

3. Sutter T, He X, Dimitrov P, Xu L, Narasimhan G, George EO, Sutter CH, Grubbs C, Savory R, Stephan-Gueldner M, Kreder D, Taylor MJ, Lubet R, Patterson TA, Kensler TW: Multiple comparisons model-based clustering and ternary pattern tree numerical display of gene response to treatment: procedure and application to the preclinical evaluation of chemopreventive agents. Mol Cancer Ther 2002, 1:1283-1292.

4. Hulshizer R, Blalock EM: Post hoc pattern matching: assigning significance to statistically defined expression patterns in single channel microarray data. BMC Bioinformatics 2007, 8:240.

doi:10.1186/1471-2105-14-S17-A20

Cite this article as: Vo and Phan: Using partially ordered sets to represent and predict true patterns of gene response to treatments. BMC Bioinformatics 2013 14(Suppl 17):A20.

\footnotetext{
* Correspondence: vphan@memphis.edu

Department of Computer Science, The University of Memphis, Memphis, TN 38152, USA
}

(c) 2013 Vo and Phan; licensee BioMed Central Ltd. This is an Open Access article distributed under the terms of the Creative Commons 